CLINICAL STUDY

\title{
Efficacy and safety of LB03002, a once-weekly sustained-release human GH for 12-month treatment in Korean children with GH deficiency
}

Jin Soon Hwang, Hae Sang Lee, Woo Yeong Chung ${ }^{1}$, Heon-Seok Han ${ }^{2}$, Dong-Kyu Jin ${ }^{3}$, Ho-Seong Kim ${ }^{4}$, Cheol-Woo Ko ${ }^{5}$, Byung-Churl Lee ${ }^{6}$, Dae-Yeol Lee ${ }^{7}$, Kee-Hyoung Lee ${ }^{8}$, Jeh-Hoon Shin ${ }^{9}$, Byung-Kyu Suh ${ }^{10}$, Han-Wook Yoo ${ }^{11}$, Hyi-Jeong Ji ${ }^{12}$, Jin-Hwa Lee ${ }^{12}$, Yoon Ju Bae ${ }^{12}$, Duk-Hee Kim ${ }^{13}$ and Sei Won Yang ${ }^{14}$

Department of Pediatrics, Ajou University School of Medicine, Suwon, Republic of Korea, ${ }^{1}$ Department of Pediatrics, College of Medicine, Inje University, Busan, Republic of Korea, ${ }^{2}$ Department of Pediatrics, College of Medicine and Medical Research Institute, Chungbuk National University, Cheongju, Republic of Korea, ${ }^{3}$ Department of Pediatrics, Sungkyunkwan University School of Medicine, Samsung Medical Center, Seoul, Republic of Korea, ${ }^{4}$ Department of Pediatrics, College of Medicine, Institute of Endocrinology, Yonsei University, Seoul, Republic of Korea, ${ }^{5}$ Department of Pediatrics, Kyungpook National University Hospital, Daegu, Republic of Korea, ${ }^{6}$ Department of Pediatrics, College of Medicine, The Catholic University of Korea, Seoul, Republic of Korea, ${ }^{7}$ Department of Pediatrics, Chonbuk National University Medical School, Research Institute of Clinical Medicine, Jeonju, Republic of Korea, ${ }^{8}$ Department of Pediatrics, College of Medicine, Korea University, Seoul, Republic of Korea, ${ }^{9}$ Department of Pediatrics, College of Medicine, Hanyang University, Seoul, Republic of Korea, ${ }^{10}$ Department of Pediatrics, College of Medicine, Seoul Saint Mary's Hospital, The Catholic University of Korea, Seoul, Republic of Korea, ${ }^{11}$ Department of Pediatrics, Asan Medical Center Children's Hospital, University of Ulsan College of Medicine, Seoul, Republic of Korea, ${ }^{12}$ LG Life Sciences, Ltd., Seoul, Republic of Korea, ${ }^{13}$ Department of Pediatrics, Sowha Children's Hospital, Seoul, Republic of Korea, ${ }^{14}$ Department of Pediatrics, College of Medicine, Seoul National University, Seoul, Republic of Korea

(Correspondence should be addressed to S W Yang; Email: growth@snu.ac.kr)

\begin{abstract}
Purpose: The purpose of this study was to investigate the efficacy and safety of LB03002, a sustainedrelease human GH (SR-hGH), compared with that of daily rhGH for 12 months in children with GH deficiency (GHD).

Methods: A total of 73 children with GHD were screened and 63 eligible subjects were randomized in a 1:1 ratio of LB03002 (SR-hGH) to daily rhGH treatment group. LB03002 was administered once weekly at a dose of $0.5 \mathrm{mg} / \mathrm{kg}$ while daily rhGH was administered for 6 consecutive days with equally divided doses to make a total of $0.21 \mathrm{mg} / \mathrm{kg}$ per week. Treatments were given for 12 months by s.c. injections. Injection site reactions and adverse events were investigated throughout the study period. Results: The mean (s.D.) height velocity (HV) showed a clinically significant increase after the 6-month treatment: $3.00(1.15) \mathrm{cm} /$ year at screening to $9.78(1.98) \mathrm{cm} /$ year at 6 months in the LB03002 group; $2.39(1.63) \mathrm{cm} /$ year at screening to $10.56(2.65) \mathrm{cm} /$ year at 6 months in the daily rhGH group. The increased HV at 12 months was still maintained in both the groups: 9.06 (1.63) cm/year at 12 months in the LB03002 group; $9.72(2.32) \mathrm{cm}$ /year at 12 months in the daily rhGH group. Most of the adverse drug reactions were mild and tolerable. No subjects were withdrawn due to adverse events. Conclusion: Weekly injection of LB03002 at a dose of $0.5 \mathrm{mg} / \mathrm{kg}$ per week was confirmed to have comparable efficacy to daily injection of $\mathrm{rhGH}$ at a dose of $0.21 \mathrm{mg} / \mathrm{kg}$ per week. Both formulations were well tolerated.
\end{abstract}

European Journal of Endocrinology 169 179-185

\section{Introduction}

At present, standard GH therapy is given by daily s.c. injection, which creates problems with compliance and adherence for patients, parents, and caregivers (1). Longer acting formulations of a variety of medicines have long been identified as a viable strategy for improving adherence to a medication regimen $(2,3,4)$.

LB03002 is a once-weekly sustained-release formulation of recombinant human GH manufactured using genetically modified Saccharomyces cerevisiae and contained in sodium hyaluronate microparticles, which are suspended in medium-chain triglycerides before injection (5). The pharmacokinetic and pharmacodynamic profile of LB03002 has been established in both children and adults with GH deficiency (GHD) $(6,7)$. Preliminary efficacy and safety results from studies on GH-deficient children and adults have shown LB03002 to be a suitable candidate for long-term replacement therapy in patients with $\operatorname{GHD}(8,9)$. In this study, the primary objective was to evaluate the longterm efficacy and safety of LB3002 compared with daily rhGH treatment for 12 months. 


\section{Subjects and methods}

\section{Subjects}

This study was conducted in 14 centers in South Korea in accordance with the ethical principles of Declaration of Helsinki and good clinical practice, and the protocol was approved by each institutional review board. From May 2005 to February 2007, prepubertal children aged 3 years or older were screened for study participation if their parents or legal representatives provided written informed consents. Children were determined to have GHD if peak GH levels were $<10 \mathrm{ng} / \mathrm{ml}$ in response to at least two GH stimulation tests (using clonidine, insulin, or levodopa). For those subjects who had been diagnosed with multiple pituitary hormone deficiencies or organic GHD, confirmatory GH stimulation test was exempted if they had records of previous GH stimulation test results indicating complete GHD $(<5 \mathrm{ng} / \mathrm{ml})$. The following inclusion criteria were also used to screen the children: i) open epiphyses; ii) height at or below the 3rd percentile for age and sex (not applicable if a child had organic GHD or GH treatment history); iii) height velocity (HV) at or below the 25th percentile for age and sex (reference to the chart of Tanner); iv) bone age (BA) of 10 years or below for girls and 12 years or below for boys; v) no previous GH treatment within 6 months before starting the study; vi) normal thyroid function or normal thyroid function maintained by hormonal therapy; and vii) height measurements at least 6 months apart before study initiation.

If children had any of the following conditions, they were excluded: i) causes of short stature other than GHD, such as chronic renal failure or Turner syndrome; ii) history of having resistance to GH; iii) concurrent chronic diseases such as diabetes; iv) possibility of allergic reaction or hypersensitivity to the ingredients of the study drugs; v) history of spinal irradiation; vi) active pituitary tumor or recurrence of pituitary tumor within 12 months before study initiation; vii) critically acute condition caused by complications from open heart surgery, abdominal operation, or multiple accidental injuries; viii) acute respiratory failure; or ix) treatment with other investigational drugs within 30 days before study initiation.

Of 73 screened children, 63 were randomized to receive one of the two study drugs. Fifty-eight subjects completed the 6-month treatment period and 53 subjects $(84 \%)$ completed the 12-month treatment period (Fig. 1). Of ten early withdrawn subjects, three subjects randomized to daily rhGH group never received a study drug and were excluded from all analysis subsets. The reasons for early withdrawal of those remaining seven subjects who were excluded were as follows: consent withdrawal or lost to follow-up (three subjects in LB03002 and one subject in daily rhGH), GH level $>10 \mathrm{ng} / \mathrm{ml}$ at screening (one subject in LB03002), abnormal thyroid function (one subject

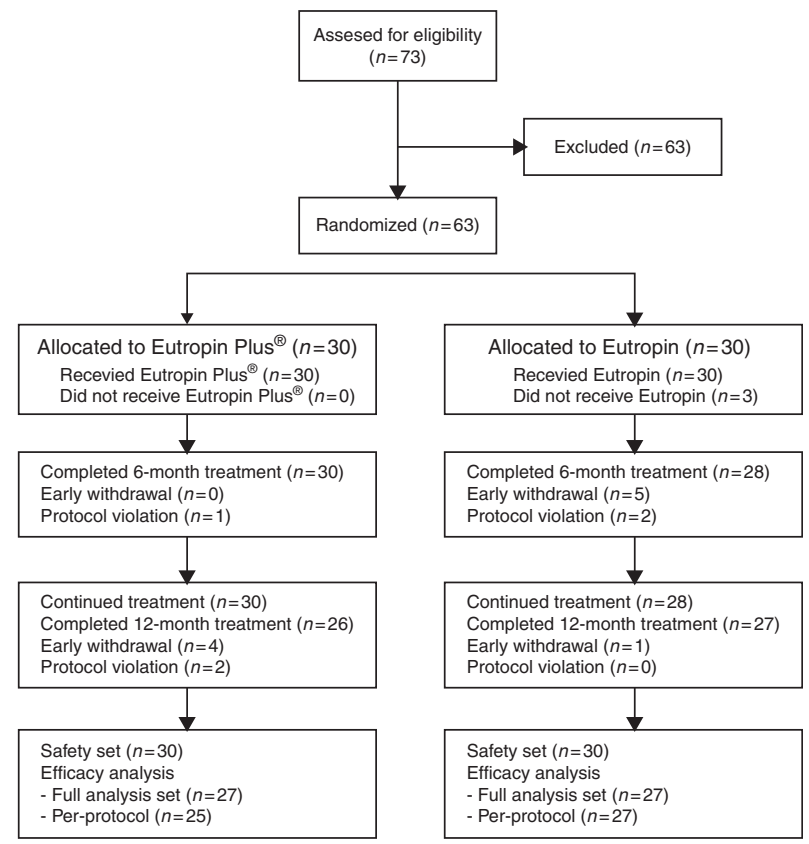

Figure 1 Subject disposition.

in daily rhGH), and advanced Tanner stage during study period (one subject in daily rhGH). One subject in LB03002 who completed the 12-month treatment but experienced advancement in Tanner stage during the study period was also excluded.

\section{Study drug}

The eligible subjects were randomized in a ratio of $1: 1$ to receive one of the two study drugs for 6 months (26 weeks): LB03002, sustained-release human GH (SR-hGH, Eutropin Plus Inj.), dosed at $0.5 \mathrm{mg} / \mathrm{kg}$ per week or daily rhGH (Eutropin) dosed at $0.21 \mathrm{mg} / \mathrm{kg}$ per week. At the end of the 6-month treatment, subjects were rolled over to a 12 -month (52 weeks) extension study to receive an additional 6-month treatment if their parents or legal representatives provided written consent. LB03002 was administered subcutaneously at a dose of $0.5 \mathrm{mg} / \mathrm{kg}$ per week once a week while a weekly dose of $0.21 \mathrm{mg} / \mathrm{kg}$ per week of daily rhGH was administered in six equally divided daily doses. The dose of LB03002 was chosen from the pharmacokinetic/ pharmacodynamic Phase II/IIIa study of LB03002. The Medical Guideline of American Association of Clinical Endocrinologists (AACE) recommended $0.3 \mathrm{mg} / \mathrm{kg}$ per week, six times a week, s.c. administration for the treatment of childhood-onset GHD patients and the dose regimen of hGH was changing to six to seven times administration a week in the field. In this study, therefore, the dose regimen of $0.21 \mathrm{mg} / \mathrm{kg}$ per week, six times a week administration was used with the purpose of obtaining approval for this dose regimen, which was a real regimen in clinics $(0.17-0.21 \mathrm{mg} / \mathrm{kg}$ 
per week, six times a week). Each vial of LB03002 and daily rhGH contained $36 \mathrm{IU}$ (12 mg) and 4 IU (1.33 mg) of hGH respectively. The study drug, prepared in appropriate volume for each subject after reconstituting with $0.6 \mathrm{ml}$ of diluents-MCT for LB03002 and sterile water for daily rhGH respectively, was administered subcutaneously on upper arms or hip region with rotating the injection site. The individual dose was adjusted every 3 months according to the change of body weight. Parents or legal representatives of subjects who performed injections recorded the administered doses and the number of used vials on the provided diary. The remaining drugs in the opened vials after use were not to be reused; thus, used vials were returned to the investigators at the next study visit. All study drugs were manufactured and provided by LG Life Sciences, Ltd.

\section{Study procedures and assay methods}

Subjects were requested to visit the study center four times after randomization visit for measurement of their height and weight. BA was estimated from an X-ray of left wrist (anterior-posterior view) according to Greulich \& Pyle method every 6 months. Measurements of IGF1, IGF binding protein-3 (IGFBP3), and thyroid function test as well as hematology and serum chemistry were performed at every visit to the clinic. Analysis of antibodies against $\mathrm{GH}$ was performed at screening, 6-month visit, and 12-month visit. Samples for measurement of IGF1 and IGFBP 3 were sent to the Seoul Clinical Laboratories (Seoul, Korea) and analyzed using DSL-5600 ACTIVE IGF1-coated tube IRMA kit (Diagnostic Systems Laboratories, Webster, TX, USA) and DSL-5600 ACTIVE IGFBP3-coated tube IRMA kit (Diagnostic Systems Laboratories) respectively. Samples for measurement of antibodies to $\mathrm{GH}$ were sent to the Institute of Laboratory Medicine of University of Leipzig (Germany) and analyzed using the radioprecipitation assay method. The IGF1 and IGFBP3 SDS for age and gender was calculated according to normal IGF1 and IGFBP3 levels for age and gender in Korea. Individual IGF1 and IGFBP3 SDS values were calculated with the formula: ((IGF1-mean)/s.D.) whereas mean and s.D. were obtained from reference (10). Adverse events were collected throughout the study period. Injection site reactions were evaluated from the recordings in the subject diaries: swelling and redness were graded as mild, moderate, and severe; warmth was graded as mild and moderate; and pain was measured using $150 \mathrm{~mm}$ visual analogue scale.

\section{Statistical analysis}

The primary objective of this study was to demonstrate the efficacy of LB03002 compared with daily rhGH in terms of between-group difference in the HV after 1-year treatment. The minimum number needed to achieve $80 \%$ power at $5 \%$ level of significance (one-sided) was
26; thus, 32 subjects in each group were to be enrolled considering 20\% drop-out rate. Efficacy evaluation was primarily based on the results of per-protocol set comprising subjects who completed the study without major protocol violations, and it was also carried out with full analysis set comprising all subjects who were dosed at least once and had posttreatment efficacy data. Safety analysis was performed with data from all subjects who were dosed at least once and had posttreatment safety data. For continuous variables, between-group difference was tested using $t$-test and difference within a group was tested using paired $t$-test; for categorical variables, between-group difference was tested using $\chi^{2}$ test or Fisher's exact test, and difference within a group was tested using McNemar test. All statistical analysis was performed using SAS version 9.1.3 (SAS Institute, Inc., Cary, NC, USA). All tests used $\alpha=0.05$ as the level of significance. The mean is presented with S.D. and median is presented with range (min, max).

\section{Results}

\section{Characteristics of study subjects}

Clinical characteristics of both groups are shown in Table 1. Distribution of age, sex, weight, and baseline auxological data was similar between the groups (Table 1). Thirty-six subjects had organic GHD; of these, 28 (78\%) had been diagnosed with craniopharyngioma, two with empty sella syndrome, one with septo-optic dysplasia, and the remaining five with other pathological lesions. Thirteen subjects (43\%) in each group had hGH treatment history with median (range) treatment duration as follows: 427 (35-1925) days in the LB03002 group and 488 (31-2067) days in the daily rhGH group. All subjects except one subject in the LB03002 group had <10 ng/ml of peak GH level to $\mathrm{GH}$ provocation tests. BA lagged chronological age (CA) by 2-3 years.

\section{Annualized HV}

Our analysis showed a clinically significant increase in mean HV in both the groups after 6-month treatment: $3.00(1.15) \mathrm{cm} /$ year at screening to 9.78 (1.98) $\mathrm{cm} /$ year at 6 months in the LB03002 group; 2.39 (1.63) $\mathrm{cm} /$ year at screening to $10.56(2.65) \mathrm{cm} /$ year at 6 months in the daily rhGH group. The increased $\mathrm{HV}$ at 12 months was still maintained in both the groups (Fig. 2): $9.06(1.63) \mathrm{cm} /$ year at 12 months in the LB03002 group; $9.72(2.32) \mathrm{cm} /$ year at 12 months in the daily rhGH group. There was no significant between-group (LB03002 - daily rhGH) difference in $\mathrm{HV}$ change (posttreatment-pretreatment). 
Table 1 Baseline demographic and auxological data. Data from full analysis set.

\begin{tabular}{|c|c|c|c|}
\hline Demographic characteristic & $\begin{array}{l}\text { LB03002 } 0.5 \mathrm{mg} / \mathrm{kg} \\
\text { per week }(n=30)\end{array}$ & $\begin{array}{l}\text { Daily rhGH } 0.21 \mathrm{mg} / \mathrm{kg} \\
\text { per week }(n=30)\end{array}$ & $P$ value \\
\hline $\operatorname{Sex}(M / F)$ & $20 / 10$ & $18 / 12$ & $0.7892^{*}$ \\
\hline \multicolumn{4}{|l|}{ Chronological age (years) } \\
\hline Mean (s.D.) & $9.05(2.55)$ & $9.25(2.46)$ & \multirow[t]{2}{*}{0.7585} \\
\hline Median (range) & $4.33-13.08$ & $4.17-15.42$ & \\
\hline \multicolumn{4}{|l|}{ Bone age (years) } \\
\hline Mean (S.D.) & $7.21(1.79)$ & $6.92(1.78)$ & \multirow[t]{2}{*}{$0.5366^{\dagger}$} \\
\hline Median (range) & $7.00(3.50,9.50)$ & $8.00(3.50,9.50)$ & \\
\hline \multicolumn{4}{|l|}{ Height SDS } \\
\hline Mean (S.D.) & $-1.80(1.15)$ & $-2.09(1.25)$ & \multirow[t]{2}{*}{0.3676} \\
\hline Median (range) & $-1.96(-4.21,0.32)$ & $-2.31(-4.45,0.48)$ & \\
\hline \multicolumn{4}{|l|}{ Weight SDS } \\
\hline Mean (S.D.) & $-0.96(1.49)$ & $-0.70(1.43)$ & \multirow[t]{2}{*}{0.4839} \\
\hline Median (range) & $-1.05(-3.27,1.72)$ & $-0.77(-3.78,2.01)$ & \\
\hline \multicolumn{4}{|l|}{ Peak $\mathrm{GH}$ response $(\mathrm{ng} / \mathrm{ml})^{\mathrm{a}}$} \\
\hline Mean (s.D.) & $2.78(3.79)$ & $1.44(2.08)$ & \multirow[t]{2}{*}{$0.0950^{\dagger}$} \\
\hline Median (range) & $1.60\left(0.02,16.90^{\mathrm{b}}\right)$ & $0.57(0.02,7.10)$ & \\
\hline \multicolumn{4}{|l|}{ Height velocity (cm/year) } \\
\hline Mean (S.D.) & $2.92(1.28)$ & $2.48(1.68)$ & \multirow[t]{2}{*}{$0.2594^{*}$} \\
\hline Median (range) & $3.15(0.40,5.00)$ & $2.90(0.00,5.40)$ & \\
\hline Idiopathic GHD/organic GHD & $12 / 18$ & $12 / 18$ & $1.0000^{*}$ \\
\hline Previous $\mathrm{GH}$ treatment $(n)^{\mathrm{c}}$ & 13 & 13 & $1.0000^{*}$ \\
\hline
\end{tabular}

\section{Secondary efficacy results}

The results for height SDS for $\mathrm{CA}$ and $\mathrm{BA}$, bone maturation, IGF1, and IGFBP 3 are presented in Table 2. Height SDS for both CA and BA significantly increased $(P<0.0001)$ at 12 months but without accelerating bone maturation, showing the bone maturation index in both groups $<1$. Significant increases in the levels of posttreatment IGFI and IGFBP3 were observed in both the groups. There were no significant between-group differences in these secondary efficacy variables.

\section{Safety}

About $80 \%$ of subjects experienced at least one adverse event regardless of causality: $80 \%(24 / 30)$ in the LB03002 group and $83 \%(25 / 30)$ in the daily rhGH group. The most frequently reported adverse event was upper respiratory infection (URI): 15 subjects $(50 \%)$ in the LB03002 group and 23 subjects (77\%) in the daily rhGH group experienced URIs; all events were mild in intensity and not related to the study drug. Adverse drug reactions (ADRs) that were considered to be related to the study drugs were observed in 11 subjects (eight in LB03002 and three in daily rhGH). Those events were mostly mild in intensity except one case of moderate myalgia (LB03002) and resolved on its own except one case of mild weight increase (LB03002), which was later found to be temporary in nature. There were 12 serious adverse events (SAEs) reported in eight subjects: gastroenteritis, inguinal hernia repair, pyrexia (two events), pharyngotonsillitis, pneumonia, and calculus bladder were reported in five subjects in the LB03002 group; convulsion (two events), epilepsy, gastroenteritis rotavirus, and pneumonia were reported in three subjects in the daily rhGH group. All SAEs were evaluated as 'not related to the study drug' and resolved with appropriate treatment. No deaths or SAEs related to the study drug occurred during the 12-month treatment period. In case of injection site reactions based on the diary data, pain was repeatedly recorded in

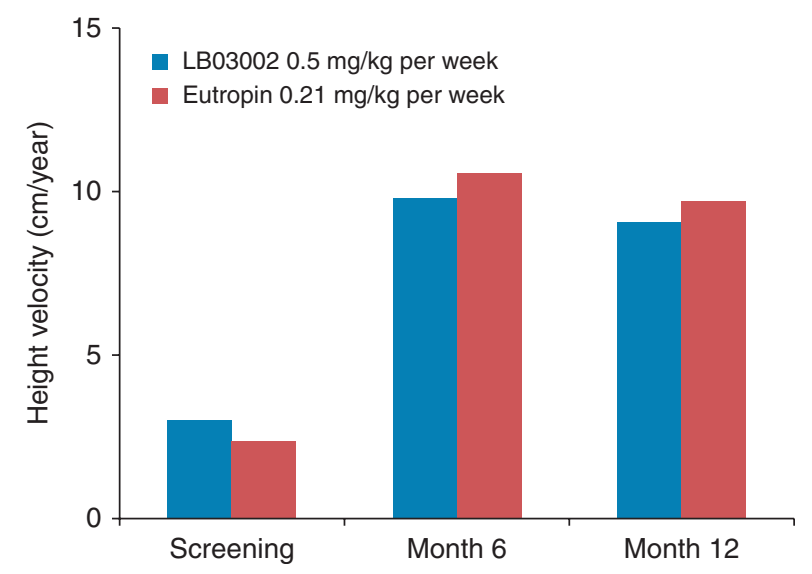

Figure 2 Height velocity estimated at screening through 12 months of treatment with either LB03002 (Eutropin Plus) or Eutropin. 
Table 2 Secondary efficacy results. Data from per-protocol set. Values are median (min, max).

\begin{tabular}{|c|c|c|c|}
\hline & $\begin{array}{l}\text { LB03002 } 0.5 \mathrm{mg} / \mathrm{kg} \\
\text { per week }(n=25)\end{array}$ & $\begin{array}{c}\text { Daily rhGH } 0.21 \mathrm{mg} / \mathrm{kg} \\
\text { per week }(n=27)\end{array}$ & $P$ value \\
\hline \multicolumn{4}{|l|}{ Height SDS CA } \\
\hline Before treatment & $-1.48(-3.89,0.53)$ & $-2.09(-3.50,0.84)$ & $0.2087^{\dagger}$ \\
\hline 6 months & $-1.07(-2.78,1.00)$ & $-1.07(-3.52,0.59)$ & $0.5346^{\dagger}$ \\
\hline 12 months & $-0.82(-2.63,1.40)$ & $-0.75(-2.84,1.07)$ & $0.3382^{\dagger}$ \\
\hline \multicolumn{4}{|l|}{ Height SDS BA } \\
\hline Before treatment & $-0.26(-2.53,3.02)$ & $0.29(-3.21,3.65)$ & $0.4976^{\dagger}$ \\
\hline 6 months & $0.14(-2.45,3.80)$ & $0.74(-2.57,3.36)$ & $0.2749^{\dagger}$ \\
\hline 12 months & $0.60(-2.83,4.43)$ & $0.66(-1.97,4.22)$ & $0.7499^{\dagger}$ \\
\hline \multicolumn{4}{|l|}{$\Delta \mathrm{BA}: \Delta \mathrm{CA}$ ratio } \\
\hline 6 months & $0.47(-0.97,1.93)$ & $0.47(-1.92,1.99)$ & $0.9571^{\dagger}$ \\
\hline 12 months & $0.49(-0.49,1.97)$ & $0.75(-0.32,2.90)$ & $0.1924^{\dagger}$ \\
\hline \multicolumn{4}{|l|}{ IGF1 SDS } \\
\hline Before treatment & $-1.5(-2.98,2.63)$ & $-1.83(-3.20,2.63)$ & $0.3984^{*}$ \\
\hline 6 months & $2.87(-1.69,9.74)$ & $1.43(-2.18,12.11)$ & $0.1347^{*}$ \\
\hline 12 months & $2.46(-2.31,10.9)$ & $1.08(-3.15,16.82)$ & $0.1716^{*}$ \\
\hline \multicolumn{4}{|l|}{ IGFBP3 SDS } \\
\hline Before treatment & $-0.73(-3.88,0.78)$ & $-1.85(-4.71,1.09)$ & $0.5011^{*}$ \\
\hline 6 months & $0.49(-0.92,2.21)$ & $-0.03(-1.81,3.18)$ & $0.0870^{\dagger}$ \\
\hline 12 months & $0.17(-2.98,2.56)$ & $0.27(-4.40,3.80)$ & $0.6880^{\dagger}$ \\
\hline
\end{tabular}

the diaries of subjects who received daily rhGH while it disappeared gradually in subjects who received LB03002. Other local reactions such as warmth, swelling, and redness were more frequently reported in the LB03002 group than in the daily rhGH group; however, these reactions were usually mild, resolved in 5 days, and occurred infrequently. The occurrence rate of these reactions was slightly higher with LB03002 on the first day of injection; however, those reactions disappeared by the time the next injection was given. There was a decreasing tendency in the frequency of injection site reactions with repeated administration in both the groups (Table 3 ).

There were no clinically relevant findings with glucose, HbA1c, thyroid function test, or lipid profile (total cholesterol, triglyceride, HDL-cholesterol), except significant increases in phosphate and alkaline phosphatase (ALP), and total cholesterol more than
$200 \mathrm{mg} / \mathrm{dl}$ in 15 subjects (25\%). There was no difference between treatment groups for the number of subjects with abnormal laboratory values in hematology, clinical chemistry, glucose homeostasis, and lipid profile.

One of $30(3 \%)$ subjects in the LBO3002 group was anti-hGH antibody positive during the 12-month treatment, while 13 of $30(43 \%)$ subjects in the daily rhGH group were anti-hGH antibody positive. Seven of 13 antibody-positive subjects in the daily rhGH group had a history of hGH treatment, and three were already positive before treatment with the study drug. Although the number of antibody-positive patients was not large enough for the statistical analysis, the exploratory subgroup analysis performed on major efficacy variables such as HV, height SDS, bone maturation, and IGF1 by antibody formation indicated that growth seemed not to be attenuated by the presence of these antibodies (data not presented).

Table 3 Number of subjects experiencing each local reaction.

\begin{tabular}{|c|c|c|c|c|c|c|}
\hline & \multicolumn{3}{|c|}{ LB03002 0.5 mg/kg per week } & \multicolumn{3}{|c|}{ Daily rhGH 0.21 mg/kg per week } \\
\hline & Week 1 & Week 13 & Week 26 & Week 1 & Week 13 & Week 26 \\
\hline Warmth & $n=28$ & $n=24$ & $n=21$ & $n=29$ & $n=22$ & $n=23$ \\
\hline Mild & 6 & 4 & 2 & 2 & 0 & 1 \\
\hline Moderate & 0 & 0 & 0 & 0 & 0 & 0 \\
\hline Erythema & $n=28$ & $n=23$ & $n=21$ & $n=29$ & $n=21$ & $n=23$ \\
\hline Mild & 9 & 4 & 3 & 2 & 1 & 1 \\
\hline Moderate & 5 & 1 & 0 & 1 & 0 & 0 \\
\hline Severe & 0 & 1 & 0 & 0 & 0 & 0 \\
\hline Swelling & $n=28$ & $n=23$ & $n=21$ & $n=29$ & $n=21$ & $n=23$ \\
\hline Mild & 8 & 5 & 4 & 4 & 1 & 1 \\
\hline Moderate & 2 & 2 & 2 & 1 & 1 & 0 \\
\hline Severe & 1 & 1 & 0 & 1 & 0 & 0 \\
\hline
\end{tabular}

$n$, number of subjects whose diary was completed for each solicited local reaction during the first week of injection at every visit. 


\section{Discussion}

This study investigated the efficacy and safety of weekly injection of $0.5 \mathrm{mg} / \mathrm{kg}$ per week LB03002 compared with daily injection of rhGH dosed at $0.21 \mathrm{mg} / \mathrm{kg}$ per week in 63 children with GHD in Korea. HV significantly increased with both treatments: $3.00 \mathrm{~cm} /$ year $(1.15 \mathrm{~cm} /$ year $)$ at screening to $9.78 \mathrm{~cm} /$ year $(1.98 \mathrm{~cm} /$ year $)$ at 6 months in the LB03002 group; $2.39 \mathrm{~cm} /$ year $(1.63 \mathrm{~cm} /$ year $)$ at screening to $10.56 \mathrm{~cm} /$ year $(2.65 \mathrm{~cm} /$ year $)$ at 6 months in the daily rhGH group, and increased $\mathrm{HV}$ at 12 months was still maintained in both the groups. The between-group difference in HV both at 6 and 12 months showed that there was no significant difference in the efficacy of both treatments in terms of accelerating growth rate.

HV result $(9 \mathrm{~cm} /$ year after 12 -month treatment) observed in this study was higher than that obtained in another study using Nutropin Depot, where HV increased to $7.9 \mathrm{~cm} /$ year after 1 year of treatment with Nutropin Depot (11). In a recent study, Peter et al. (8) reported that the first-year HV was $\sim 12 \mathrm{~cm} /$ year for the 0.5 and $0.7 \mathrm{mg} / \mathrm{kg}$ per week LB03002 dose in children with GHD.

Significant increases $(P<0.05)$ in height SDS both for $\mathrm{CA}$ and BA, IGF1, and IGFBP 3 were also observed, which complemented the efficacy results of the annualized HV. Bone maturation rate (change in BA divided by change in CA) remained $<1.0$, which indicated that $\mathrm{GH}$ treatment did not advance BA. In our results, some IGF1 SDS data had high values that are summarized in Table 2. We thought that these results could be partly attributed to methodological differences between our study and reference data as well as relatively small sample size in reference (10). IGF1 levels vary among laboratories because assay methods are not standardized. IGF1 levels were also affected by other factors such as nutrition (12).

Increased phosphate and ALP were observed during the study and it was interpreted as an indirect result of accelerated growth induced by study treatments. As the activity of ALP and phosphate level are highest at the time of maximum osteoblastic activity occurring with bone growth, it is generally 1.5 - to 2.5 -fold higher in children than in the normal adult population.

Formation of anti-hGH antibodies was detected in some patients but was not considered to be of clinical relevance and was in line with previous reports on treatment with daily GH preparation of the same origin as the GH used for the LB03002 sustained-release formulation. The growth rate did not seem to be affected by the antibody.

Local injection site reactions are unavoidable with the injection of large molecules such as hyaluronic acid; however, the injection site reactions reported in this study were mild and tolerable enough to be bearable by young children. Injection site reactions were similarly reported as adverse events from both the treatment groups.

It was noted that some of the subjects had total cholesterol $>200 \mathrm{mg} / \mathrm{dl}$. About half of these subjects had a history of surgery for craniopharyngioma. The total cholesterol level in these subjects did not change much during treatment with the study drugs. As GH contributes to lipolysis of adipose tissue, increased total cholesterol and LDL with decreased HDL might be accompanied with conditions of GHD. There has been a report that $25-75 \%$ of children who had a history of surgery for craniopharyngioma were overweight. Except for these findings, there was no notable change in laboratory tests.

In conclusion, 6-month annualized $\mathrm{HV}$ data of this study demonstrated that weekly injection of LB03002 at a dose of $0.5 \mathrm{mg} / \mathrm{kg}$ per week was comparable to daily injection (for 6 days/week) of rhGH at a divided dose of $0.21 \mathrm{mg} / \mathrm{kg}$ per week in increasing growth rate of prepubertal GHD patients. The actual HV at 12 months was confirmed to be comparable between LB03002 and daily rhGH. The safety of LB03002 was also well tolerated.

\section{Declaration of interest}

None of the authors have any conflict of interest to disclose.

\section{Funding}

Financial support for this study was provided by LG Life Sciences, Ltd., Republic of Korea. H-J Ji, J-H Lee, and Y J Bae are employed by LG Life Sciences. J S Hwang, W Y Chung, D-K Jin, C-W Ko, H-W Yoo, D-H Kim, and S W Yang received consulting fees from LG Life Sciences, Ltd., Republic of Korea.

\section{Acknowledgements}

The authors thank Hye-jin Shin and Hyunjoo Kim for their assistance in carrying out the study and John Kim for the initiation and the operation management of the study. They also appreciate Dr Ji Yeon Lim for her significant contribution to the protocol preparation and data analysis with her statistical point of view.

\section{References}

1 Rosenfeld RG \& Bakker B. Compliance and persistence in pediatric and adult patients receiving growth hormone therapy. Endocrine Practice 200814 143-154. (doi:10.4158/EP.14.2.143)

2 Polonsky WH, Fisher L, Hessler D, Bruhn D \& Best JH. Patient perspectives on once-weekly medications for diabetes. Diabetes, Obesity $\mathcal{E}$ Metabolism 201113 144-149. (doi:10.1111/j.14631326.2010.01327.x)

3 Kruk ME \& Schwalbe N. The relation between intermittent dosing and adherence: preliminary insights. Clinical Therapeutics $2006 \mathbf{2 8}$ 1989-1995. (doi:10.1016/j.clinthera.2006.12.011)

4 Osterberg L \& Blaschke T. Adherence to medication. New England Journal of Medicine 2005353 487-497. (doi:10.1056/ NEJMra050100)

5 Peterkova V, Arslanoglu I, Bolshova-Zubkovskaya E, Romer T, Zdravkovic D, Kratzsch J, Ji HJ, Savoy C \& Saenger P. A randomized, 
double-blind study to assess the efficacy and safety of valtropin, a biosimilar growth hormone, in children with growth hormone deficiency. Hormone Research 200768 288-293. (doi:10.1159/ 000105494)

6 Peter F, Savoy C, Ji HJ, Juhasz M, Bidlingmaier M \& Saenger P. Pharmacokinetic and pharmacodynamic profile of a new sustained-release GH formulation, LB03002, in children with GH deficiency. European Journal of Endocrinology $2009 \mathbf{1 6 0}$ 349-355. (doi:10.1530/EJE-08-0703)

7 Bidlingmaier M, Kim J, Savoy C, Kim MJ, Ebrecht N, de la Motte S \& Strasburger CJ. Comparative pharmacokinetics and pharmacodynamics of a new sustained-release growth hormone $(\mathrm{GH})$, LB03002, vs daily GH in adults with GH deficiency. Journal of Clinical Endocrinology and Metabolism 200691 2926-2930. (doi:10.1210/jc.2006-0514)

8 Peter F, Bidlingmaier M, Savoy C, Ji HJ \& Saenger PH. Three-year efficacy and safety of LB03002, a once-weekly sustained-release growth hormone $(\mathrm{GH})$ preparation, in prepubertal children with GH deficiency (GHD). Journal of Clinical Endocrinology and Metabolism 201297 400-407. (doi:10.1210/jc.2011-2234)

9 Biller BM, Ji HJ, Ahn H, Savoy C, Siepl EC, Popovic V, Coculescu M, Roemmler J, Gavrila C, Cook DM et al. Effects of once-weekly sustained-release growth hormone: a double-blind, placebo- controlled study in adult growth hormone deficiency. Journal of Clinical Endocrinology and Metabolism 201196 1718-1726. (doi:10.1210/jc.2010-2819)

10 Hyun SE, Lee BC, Suh BK, Chung SC, Ko CW, Kim HS, Lee KH, Yang SW, Shin CH, Hwang JS et al. Reference values for serum levels of insulin-like growth factor-I and insulin-like growth factor binding protein-3 in Korean children and adolescents. Clinical Biochemistry 201245 16-21. (doi:10.1016/j.clinbiochem.2011. 10.003)

11 Reiter EO, Attie KM, Moshang T Jr, Silverman BL, Kemp SF, Neuwirth RB, Ford KM \& Saenger P. A multicenter study of the efficacy and safety of sustained release GH in the treatment of naive pediatric patients with GH deficiency. Journal of Clinical Endocrinology and Metabolism $2001 \quad 86 \quad 4700-4706$. (doi:10.1210/jc.86.10.4700)

12 Thissen JP, Ketelslegers JM \& Underwood LE. Nutritional regulation of the insulin-like growth factors. Endocrine Reviews 199415 80-101.

Received 21 February 2013

Revised version received 3 May 2013

Accepted 16 May 2013 